\title{
Performance Evaluation of Balancing Bicopter using P, PI, and PID Controller
}

\author{
Esa Apriaskar ${ }^{1}$, Fahmizal $^{2}$, Nur Azis Salim ${ }^{3}$, and Dhidik Prastiyanto ${ }^{4}$ \\ ${ }^{1,3,4}$ Department of Electrical Engineering, Universitas Negeri Semarang \\ Gunungpati, Semarang, 50229, Indonesia \\ ${ }^{2}$ Department of Electrical Engineering and Informatics, Vocational College, Universitas Gadjah Mada \\ Jl. Yacaranda, Sekip Unit IV, Yogyakarta, 55281, Indonesia \\ esa.apriaskar@mail.unnes.ac.id ${ }^{1}$,fahmizal@ugm.ac.id ${ }^{2}$,nurazissalim@mail.unnes.ac.id ${ }^{3}$, \\ dhidik.prastiyanto@mail.unnes.ac.id ${ }^{4}$
}

\begin{abstract}
Due to potential features of unmanned aerial vehicles for society, the development of bicopter has started to increase. This paper contributes to the development by presenting a performance evaluation of balancing bicopter control in roll attitude. It aims to determine the best controller structure for the balancing bicopter. The controller types evaluated are based on Ziegler-Nichols tuning method; they are proportional (P), proportional-integral (PI), and proportional-integral-derivative (PID) controllers. Root locus plot of the closed-loop balancing bicopter system is used to decide the tuning approach. This work considers a difference in pulse-width-modulation (PWM) signal between the left and right rotors as the signal control and bicopter angle in roll movement as the output. Parameters tuned by the method are $\mathrm{Kp}$, Ti, and Td which is based on the ideal PID structure. The performance test utilizes rising time, settling time, maximum overshoot, and steady-state error to determine the most preferred controller. The result shows that PIcontroller has the best performance among the other candidates, especially in maximum overshoot and settling time. It reaches 8.34 seconds in settling time and $3.71 \%$ in maximum overshoot. Despite not being the best in rising time and resembling PID-controller performances in steady-state error criteria, PIcontroller remains the most preferred structure considering the closeness of the response to the desired value.
\end{abstract}

Keywords — bicopter, roll attitude, Ziegler-Nichols, PID controller

\section{INTRODUCTION}

Flying vehicle is not a new product in the engineering field. Even so, rapid development of unmanned aerial vehicles (UAVs) has caused research in the field of flying vehicles to become a hotspot in the world today. Due to its potential features, UAVs are available to be used for many applications, such as sowing fertilizer for plants in paddy fields [1], [2], monitoring the activity of a volcano mountain [3], selflocalization, and mapping of specific environment [4], [5], video shooting purpose [6], even for surveillance vehicle in military mission [7], [8]. Therefore, it has been such a challenge for researchers to give their significant contrivances for technology development on UAVs.

Research on minimizing the number of rotors in a rotortype UAV has been one of challenging issues. Bicopter is one of the examples which has only two rotors. It has an advantage in terms of flight time compared to other common rotor-type UAVs which has more rotors. The reduction in the number of rotors can lead to an alleviation of unit time power demand and consequently improving flight time [9]. However, problem of controlling bicopter system is not a trivial task due to its complexity regards to mathematical dynamics model. Designed controller has to be capable for stabilizing bicopter with acceptable response time and overcoming oscillation effect at the same time to maintain the desired trajectory.
Controllers based on PID (Proportional, Integral, and Derivative) components tend to be appropriate ways for controlling bicopter. Proportional term has a role to improve response time of bicopter performance, while derivative term can provide a damping effect for unwanted overshoots. In order to assure bicopter performing desired movement, integral term may eliminate steady-state error. Several works regarding bicopter control have also involved PID components. However, they are mostly using trial and error to determine the control parameters. In [9], PID controller was presented to stabilize the attitude of bicopter in both simulation and real platform. This, however, tuned the parameters using trial and error method which could waste time due to difficulty in tuning parameter control for such a complex system. In [10], a modified N-PID controller was used to produce more stable final performances of bicopter in comparison to the standard PID controller, despite of this also used trial and error method for tuning the parameter. A more complex control problem was presented in [11]. It utilized liftpropeller gyroscopic to naturally stabilize the bicopter in a hovering position. It is considered as mechanical controller and a pilot action is utilized to help the stabilization under disturbances. It applied integral-controller to represent the pilot control. Stabilization response depends on the pilot over the controller. A different design of two-rotor aerial vehicle has also been proposed by [12]. It actually follows the concept 
of a fixed wing plane, but gives two tilt-rotors and a ductedfan. The modifications are intended to generate a lift force during a vertical flight. PID controller adjusted to the system were not tuned. It followed default parameters of the PX4autopilot device.

In this paper, Ziegler-Nichols tuning method is used to determine the control parameter based on PID components. This work also aims to evaluate which controller types better in performing attitude control of balancing bicopter system. The performance of the method is tested using a balancing bicopter on roll movement. The balancing bicopter model and the determination of control parameter for bicopter system using Ziegler-Nichols method are described in section II. Section III presents how the controllers perform through simulation. It also includes the performance comparison between P, PI, and PID-controllers tuned by Ziegler-Nichols concept. Section IV concludes the paper with suggestions for future works.

\section{DESIGN AND METHOD}

\section{A. Balancing Bicopter Model}

The input and output variables have to be determined first before controlling a system. Balancing bicopter system used in this work consider roll angle $(\varphi)$ as the output and actuating signal for two rotors as the input. Figure 1 shows the balancing bicopter design applied in this paper. Each rotor $i$ produces moment $M_{i}$ and aerodynamic force $F_{i}$. The relations between them are described in (1) - (2) with $K_{f}$ as constant for aerodynamic force and $K_{M}$ for moment constant. The angular velocity of the rotor applied in balancing bicopter are represented with $\Omega_{i}(i=1,2)$.

$$
\begin{gathered}
F_{i}=K_{f} \Omega_{i}{ }^{2} \\
M_{i}=K_{M} \Omega_{i}{ }^{2}
\end{gathered}
$$

From (1)-(2), which describe the forces and moments generated by the propellers, we obtain the moment $M_{B}$ that can lift a bicopter, which is expressed in (3). $L$ represents a range between rotation axis of the rotor to the center body of bicopter.

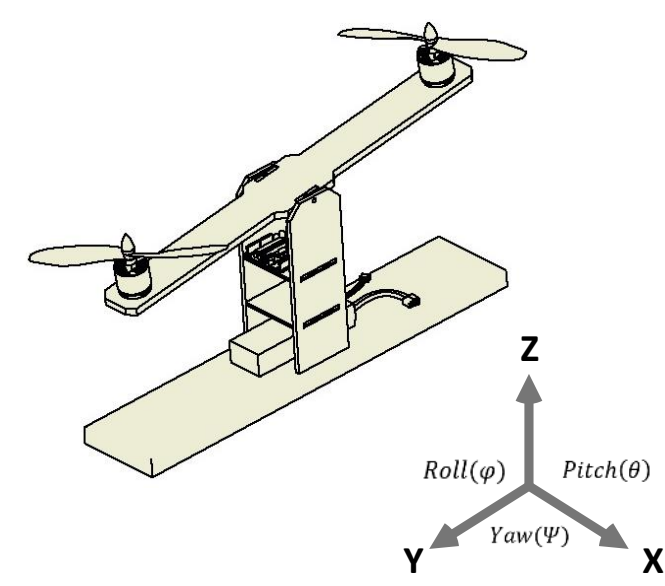

Figure 1. Balancing bicopter design

$$
M_{B}=\left[\begin{array}{c}
L K_{f}\left(\Omega_{1}^{2}-\Omega_{2}^{2}\right) \\
K_{M}\left(\Omega_{1}^{2}+\Omega_{2}^{2}\right)
\end{array}\right]
$$

To represent the dynamics of a balancing bicopter with rotor signal as the input and roll angle as the output, [13] has developed a transfer function model. Rigorous modelling steps have been introduced involving multi-level periodic perturbation signal. Several model structures were generated and validated with a certain distribution of data sets. Thus, it obtains the most appropriate model by comparing their fitness values. Final validation using a feedback control scheme has singled out a $4^{\text {th }}$-order transfer function as written in (4).

$$
\frac{\varphi_{(s)}}{\Delta P W M_{(\mathrm{s})}}=\frac{0.6126 s^{3}-1.359 s^{2}+28.81 s+5.315}{s^{4}+2.27 s^{3}+19.89 s^{2}+14.84 s+2.74}
$$

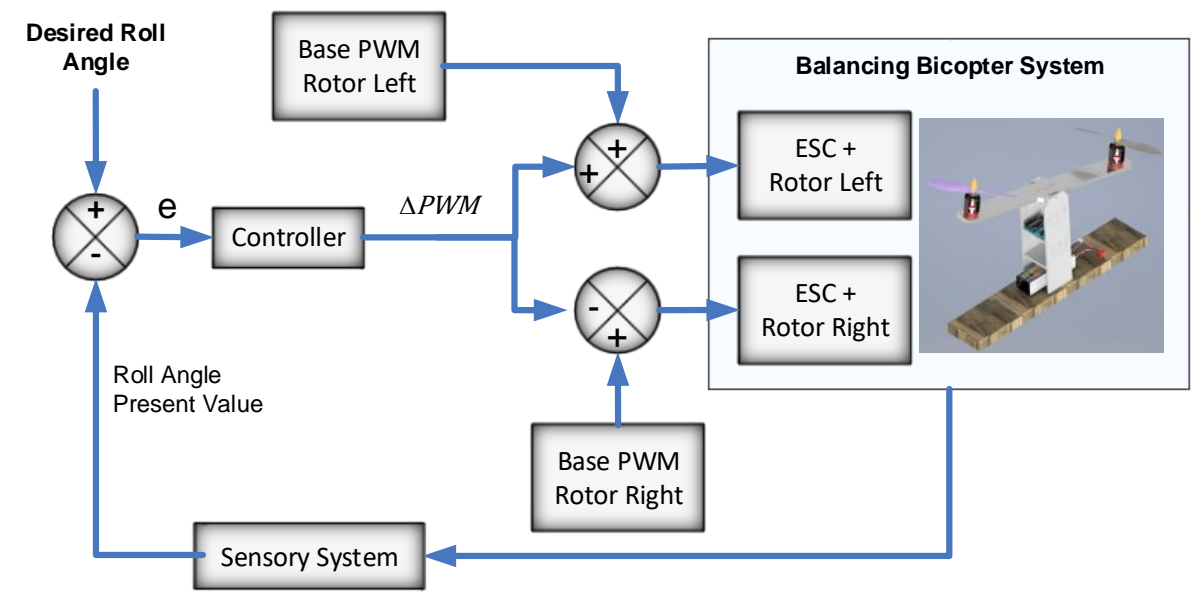

Figure 2. Diagram block for roll attitude control of balancing bicopter 
$\triangle P W M_{(\mathrm{s})}$ is the difference value of PWM (Pulse Width Modulation) signals that operate left and right actuators of the bicopter. The term $(s)$ in the input and output variables denote that the equation is in the frequency domain. This parameter also becomes manipulated variable in a closed-loop control scheme.

Figure 2 depicts block diagram of the closed-loop control scheme for the system. The measured difference between desired and roll angle present value becomes primary input of the controller. It needs sensory systems that include 3-axis accelerometer and a 3-axis gyroscope sensor inside an MPU6050 to obtain the present value. Thus, the controller generates a control signal in the form of $\triangle P W M_{(\mathrm{s})}$. It becomes an addition to each actuating signal for left and right rotors. It is considered as a base PWM signal. The base PWM signal for left and right rotors can be different from each other due to mechanical device specification tolerances. Afterward, the actuating signal drives both rotors through an electronic speed controller (ESC).

\section{B. Ziegler-Nichols based PID controller}

The controlled system dynamics are represented by a transfer function model as stated in (4). For a linear transfer function model, the PID controller can be written as (5), where $k_{p}, k_{i}$, and $k_{d}$ are proportional, integrative, and derivative constant of the controller. $e(t)$ is the error that defines the gap between the desired value and the output of the system $y(t)$ which is obtained during the controlling process. Equation (6) shows the transfer function of the PID controller after being transformed by Laplace transform [14].

$$
\begin{gathered}
u(t)=k_{p} e(t)+k_{i} \int_{0}^{t} e(\tau) d \tau+k_{d} \frac{d e(t)}{d t} \\
U(s)=\left(k_{p}+\frac{k_{i}}{s}+k_{d} s\right) e(s)
\end{gathered}
$$

There are several methods to decide appropriate PID parameters for a particular control system or so-called tuning method. One of the most well-known methods for tuning a PID controller is Ziegler-Nichols. That is classified into two methods based on the existence of integrator or dominant complex-conjugate poles [15]. The first approach is for the system that does not consist of both integrator and complexconjugate poles. It is based on an open-loop structure relying on reaction curves. The second approach is for the system which has one of integrator or complex-conjugate poles. It is based on a closed-loop structure with the computation of ultimate gain $\mathrm{Kcr}$ and ultimate period $\mathrm{Pcr}$. Since the system involved in this paper is possessing complex-conjugate poles, as shown in Figure 3, it is best to use the closed-loop approach.

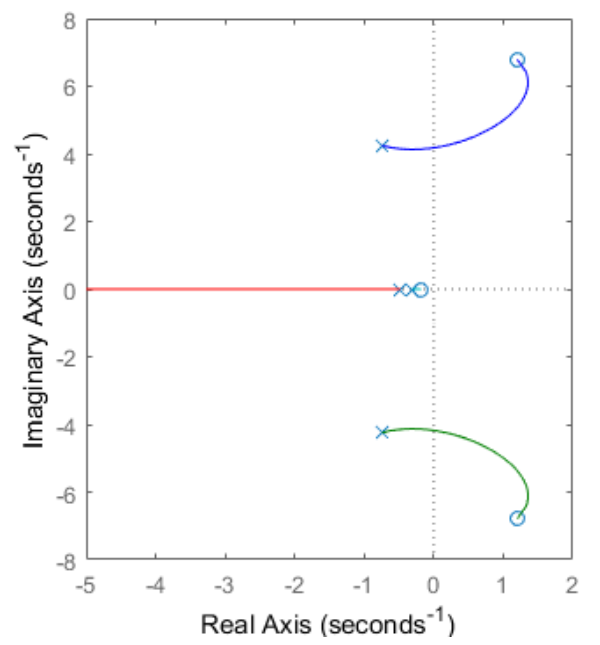

Figure 3. Root locus plot of balancing bicopter system

Ultimate gain $\mathrm{Kcr}$ is a proportional gain which creates a marginally stable condition. This condition shows a sustained oscillation of the output signal, as shown in Figure 4. Firstly, we set $k_{i}=0$ and $k_{d}=0$ and then increase the value of $k_{p}$ from 0 to critical value that first exhibits marginally stable condition. Obtaining $\mathrm{Kcr}$ can be faster with the assistance of a root locus plot. It gives information about a gain that is close to the value of $\mathrm{Kcr}$ from points that are tangent with the imaginary axis. To define Pcr, we calculate a period of the oscillation as shown in Figure 4. From our simulation, it finds $K c r=1.37$ and $P c r=0.537$. With those two variables, Ziegler-Nichols method is able to determine the appropriate parameters for $\mathrm{P}$, PI, and PID-controller scheme, as shown in Table I.

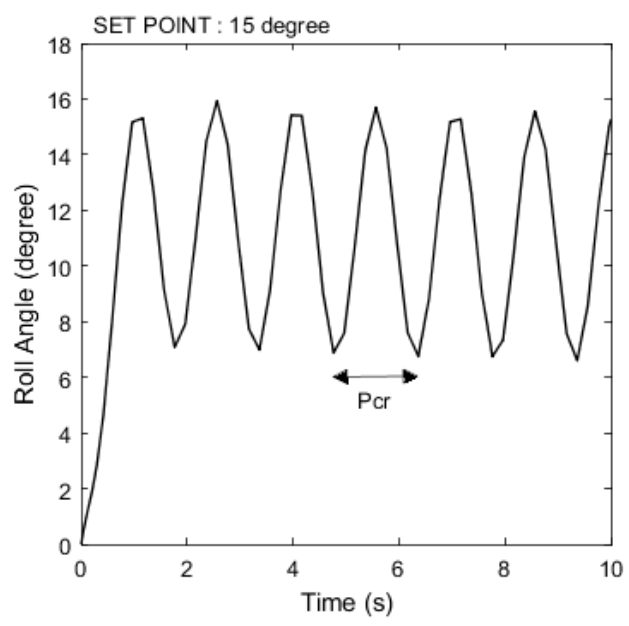

Figure 4. Marginally stable condition

TABLE I. ZIEGLER-NICHOLS RULES

\begin{tabular}{|c|c|c|c|}
\hline Controller type & $\boldsymbol{k}_{\boldsymbol{p}}$ & $\boldsymbol{T}_{\boldsymbol{i}}$ & $\boldsymbol{T}_{\boldsymbol{d}}$ \\
\hline P-controller & $0.5 \mathrm{Kcr}$ & - & - \\
\hline PI-controller & $0.45 \mathrm{Kcr}$ & $0.833 \mathrm{Pcr}$ & - \\
\hline PID-controller & $0.6 \mathrm{Kcr}$ & $0.5 \mathrm{Pcr}$ & $0.125 \mathrm{Pcr}$ \\
\hline
\end{tabular}




$$
U(s)=k_{p}\left(1+\frac{1}{T_{i} s}+T_{d} s\right) e(s)
$$

Another thing to concern about Ziegler-Nichols method is that it works with an ideal PID structure. It makes (6) is converted into (7). It notices that $k_{i}$ and $k_{d}$ are also affected by the value of $k p$. To independently tune $k_{i}$ or $k_{d}$, it is available by changing the value of time integral, $T_{i}$ or time derivative, $T_{d}$.

\section{RESULT AND DISCUSSION}

Testing performances of designed controllers can be shown through a closed-loop test respecting on the transient response. In this paper, closed-loop tests consider 15 degrees in the roll angle of the balancing bicopter as the set point. The 15-degree set point is selected randomly and considered that it is enough to move the bicopter vertically. Evaluations are conducted by comparing P, PI, and PID-controller, which of parameters are tuned using Ziegler-Nichols method. Based on the calculation mentioned in Table I, the parameter value of $k_{p}$ in P-controller is 0.685 . It generates a transient response as shown in figure 5 which indicates that P-controller is not suitable for the system due to the existence of a steady-state error. It has actually reached a stable state, but the produced error is unacceptable. Thus, an integrator is highly required to improve performance.

There are two structure options for the controller, including integrator, and they are PI-controller and PIDcontroller. Parameters utilized in PI-controller based on the Ziegler-Nichols method are $k_{p}$ and $T_{i}$ with a value of 0.6165 and 0.447, while for PID-controller, there are $k_{p}, T_{i}$, and $T_{d}$ with the value of $0.822,0.2685$, and 0.067125 , respectively.

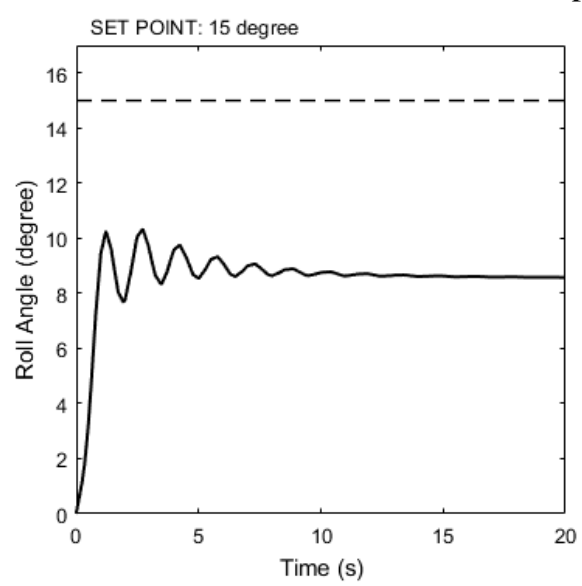

Figure 5. Closed-loop response of the system using P-controller

As seen in Figure 6 and 7, improvements have been achieved. Table II highlights a more detailed comparison performance of the three controller types. The addition of an integral part of controllers tends to be a successful step to eliminate steady-state error. PI-controller can reduce the settling time and maximum overshoot of the closed-loop system, despite slightly lacking in rise-time performance. The best in rise time criteria is when the system uses $\mathrm{P}$-controller in 0.66 seconds, but it is unacceptable due to the drawback in error steady state.
TABLE II. COMPARISON PERFORMANCE OF P, PI, AND PID-CONTROLLER

\begin{tabular}{|c|c|c|c|}
\hline Criteria & P-controller & PI-controller & PID-controller \\
\hline Rise Time (s) & 0.66 & 1.96 & 0.73 \\
\hline $\begin{array}{c}\text { Settling Time } \\
(\mathrm{s})\end{array}$ & 10.52 & 8.34 & 12.08 \\
\hline $\begin{array}{c}\text { Maximum } \\
\text { Overshoot } \\
(\%)\end{array}$ & 20.51 & 3.71 & 21.83 \\
\hline $\begin{array}{c}\text { Steady-state } \\
\text { error (degree) }\end{array}$ & 6.42 & 0 & 0 \\
\hline
\end{tabular}

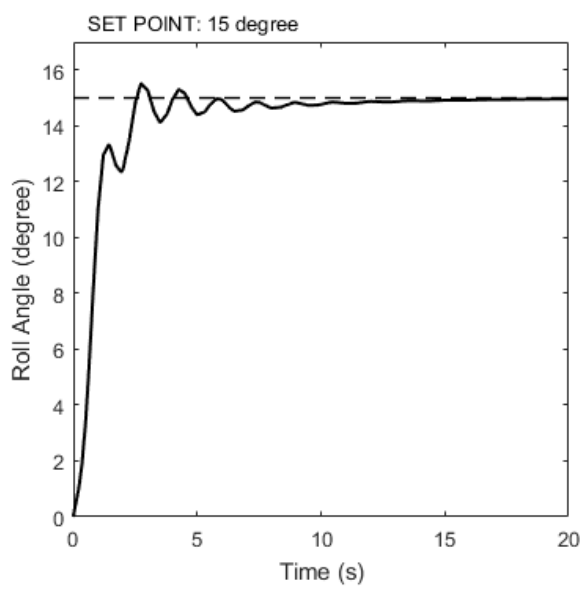

Figure 6. Closed-loop response of the system using PI-controller

Compared to PID-controller performance, rise-time performance of PI-controller is still acceptable since reducing a settling time is more important than rise time regarding the closeness of the response to the desired value [16]. PIDcontroller can quicken the rise time performance, but it gives considerable drawbacks in the other criteria, especially in the overshoot. Speeding up rise time may cost a large amount of maximum overshoot [17]. It is approximately $18 \%$ apart from PI-controller, which only possess $3.71 \%$ or equals to 0.56 degree. For settling time, PI-controller is also better than PIDcontroller in 8.34 seconds.

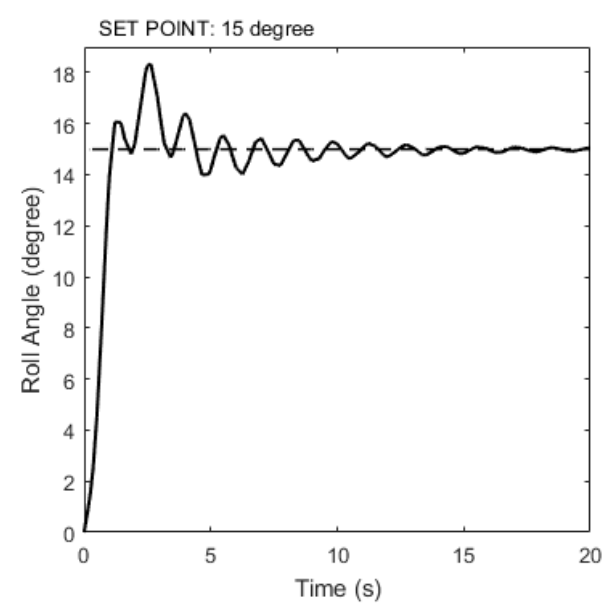

Figure 7. Closed-loop response of the system using PID-controller

The work about multi-copter attitude control conducted by [18] also uses maximum overshoot, rising time, settling time, and steady-state error to check the performance of the 
proposed controller as shown in Table II, despite not evaluating the comparative performance between P, PI, and PID-controller. It has confirmed through a simulation that the proposed controller could gain steady-state error and maximum overshoot which are close to 0 for attitude control in a quadcopter. It has also occurred with bicopter system in [9]. It resembled roll angle attitude control, which resulted in zero steady-state error and maximum overshoot. As shown in Table II, the three controllers evaluated in this work could not obtain absolutely the same performance. It declares that the Ziegler-Nichols tuning method is not enough to eliminate the overshoot in the case of balancing bicopter control.

Nevertheless, we can determine the best controller type between P, PI, and PID-controller for the balancing bicopter system. PI-controller tends to perform the best for a balancing bicopter system in roll angle attitude control based on zieglernichols tuning method. It can obtain overshoot and steadystate error which are closer to 0 rather than other controllers.

A robustness test has also been conducted to ensure that the determination of controller type is proper. An impulse disturbance can be applied to test the robustness of a controller [19]. The test considers that balancing bicopter is initially in a hovering position. Thus, it is given reference 0 degree in roll angle. An impulse signal is supplemented with 0.1 second in duration time starting from 0.25 second. Figure 8 shows the comparison response between P, PI, and PID controller after being perturbed. In this test, PID-controller has the highest stabilizing time in approximately 12 seconds. It is followed by P-controller which achieves 10.81 seconds. PIcontroller looks to give better performance than the two controllers with 8.77 seconds.

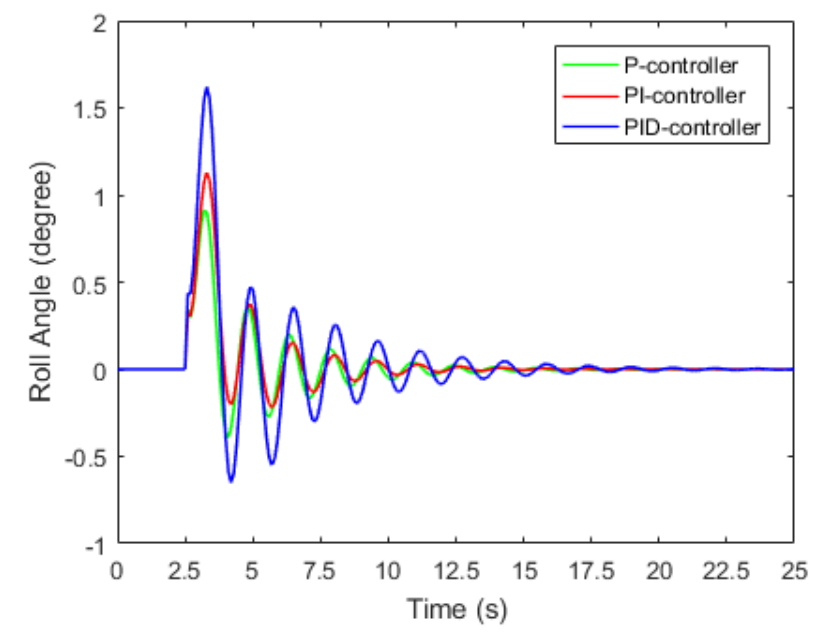

Figure 8. Robustness test using impulse disturbance for P, PI, and PID controller tuned by Ziegler-Nichols

The robustness test using impulse perturbation has further confirmed that PI-controller is the best option among the other options. It can stabilize the roll angle under an impulse disturbance faster than P and PID controller tuned by ZieglerNichols. To maximize the response, especially improving the overshoot performances, more advanced tuning methods, such as involving genetic algorithm [20], particle swarm optimization [21], iterative-tuning method [22] or another optimization approach may be necessary. It is also possible to create a more robust controller after utilizing artificial intelligence in the control parameter tuning process [23].

\section{CONCLUSION}

Finally, this work has evaluated performances of balancing bicopter control on roll movement tuned by Ziegler-Nichols concept. The closed-loop approach of Ziegler-Nichols method has been utilized due to the existence of complex-conjugate poles in the balancing bicopter transfer function. The tuning method can provide fine parameters for three controller types; they are P, PI, and PID-controllers. The result concludes that based on Ziegler-Nichols tuning method, PI-controller is the most preferred option for balancing bicopter control system among the other controller types due to better performance, especially in settling time and maximum overshoot criteria. The robustness test using impulse disturbance has also ensured the determination of PI-controller. It has produced shorter stabilizing time compared to the P and PID controller. However, it seems still possible to develop the controller parameter tuning since several works indicate that the performance of maximum overshoot can still be improved. Using a more advanced tuning method can be an unfilled space for future work in the development of a bicopter control system.

\section{REFERENCES}

[1] S. D. Kale, S. V Khandagale, S. S. Gaikwad, S. S. Narve, and P. V Gangal, "Agriculture Drone for Spraying Fertilizer and Pesticides," Int. J. Adv. Res. Comput. Sci. Softw. Eng., vol. 5, no. 12, pp. 804-807, 2015.

[2] Y. B. Huang, S. J. Thomson, W. C. Hoffmann, Y. Bin Lan, and B. K. Fritz, "Development and prospect of unmanned aerial vehicle technologies for agricultural production management," Int. J. Agric. Biol. Eng., vol. 6, no. 3, pp. 1-10, 2013.

[3] S. Amici et al., "Volcanic environments monitoring by drones, Mud Volcano case Study," Int. Arch. Photogramm. Remote Sens. Spat. Inf. Sci. Vol. XL-1/W2, 2013 UAV-g2013, vol. XL-1/W2, no. September, pp. 5-10, 2013

[4] E. Apriaskar, Y. P. Nugraha, and B. R. Trilaksono, "Simulation of Simultaneous Localization and Mapping Using Hexacopter and RGBD Camera," in International Conference on Automation, Cognitive Science, Optics, Micro Electro-Mechanical System, and Information Technology, 2017, pp. 48-53.

[5] Y. S. Chou and J. S. Liu, "A robotic indoor 3D mapping system using a 2D laser range finder mounted on a rotating four-bar linkage of a mobile platform," Int. J. Adv. Robot. Syst., vol. 10, 2013.

[6] Q. Galvane, J. Fleureau, F.-L. Tariolle, and P. Guillotel, "Automated Cinematography with Unmanned Aerial Vehicles," in WICED 2016 Proceedings of the Eurographics Workshop on Intelligent Cinematography and Editing, 2016, pp. 23-30.

[7] S. Agarwal, S. Shetty, A. Bhagat, J. Ghule, and P. S. H. Mane, "UAV based Quadcopter with Wheels," Int. J. Sci. Res. Dev., vol. 2, no. 2, pp. 1003-1006, 2014.

[8] R. K. Rangel and A. C. Terra, "Development of a Surveillance tool using UAV' s," IEEE Aerosp. Conf., no. March 2015, pp. 1-11, 2018.

[9] Q. Zhang, Z. Liu, J. Zhao, and S. Zhang, "Modeling and attitude control of Bi-copter," in AUS 2016 - 2016 IEEE/CSAA International Conference on Aircraft Utility Systems, 2016, vol. 100191, pp. 172176.

[10] L. Hrečko, J. Slačka, and M. Halás, "Bicopter stabilization based on IMU sensors," in 20th International Conference on Process Control, 2015, pp. 192-197.

[11] G. R. Gress, "Natural Pitch Stabilization of Bicopters in Hover Using Lift-Propeller Gyroscopics," J. Guid. Control. Dyn., vol. 41, no. 2, pp. 476-487, Feb. 2018. 
[12] J. M. Bustamante, C. A. Herrera, E. S. Espinoza, C. A. Escalante, S Salazar, and R. Lozano, "Design and Construction of a UAV VTOL in Ducted-Fan and Tilt-Rotor Configuration," in 2019 16th International Conference on Electrical Engineering, Computing Science and Automatic Control (CCE), 2019, pp. 1-6.

[13] Fahmizal, M. Arrofiq, E. Apriaskar, and A. Mayub, "Rigorous Modelling Steps on Roll Movement of Balancing Bicopter using Multilevel Periodic Perturbation Signals," in 6th International Conference on Instrumentation, Control, and Automation (ICA), 2019, pp. 52-57.

[14] M. Z. Fadel, M. G. Rabie, and A. M. Youssef, "Modeling, Simulation and Control of a Fly-by-wire Flight Control System Using Classical PID and Modified PI-D Controllers," J. Eur. des Systèmes Autom., vol. 52, no. 3, pp. 267-276, Aug. 2019.

[15] T. T. Hlaing, "Simulation of Ziegler-Nichols PID Tuning for Position Control of DC Servo Motor," Int. J. Sci. Res. Publ., vol. 9, no. 7, p. p9184, Jul. 2019.

[16] A. A. Aly and F. A. Salem, "A New Accurate Analytical Expression for Rise Time Intended for Mechatronics Systems Performance Evaluation and Validation," Int. J. Autom. Control Intell. Syst., vol. 1, no. 2, pp. 51-60, 2015.

[17] R. C. Dorf and R. H. Bishop, Modern Control Systems, 12th ed. New Jersey: Prentice Hall, 2011.
[18] M. Reyad, M. Arafa, and E. A. Sallam, "An optimal PID controller for a qaudrotor system based on DE algorithm," in 2016 11th International Conference on Computer Engineering \& Systems (ICCES), 2016, pp. 444-451.

[19] E. Susanto, A. Surya Wibowo, and E. Ghiffary Rachman, "Fuzzy Swing Up Control and Optimal State Feedback Stabilization for SelfErecting Inverted Pendulum," IEEE Access, vol. 8, pp. 6496-6504, 2020.

[20] Y. Qin, G. Zhao, Q. Hua, L. Sun, and S. Nag, "Multiobjective Genetic Algorithm-Based Optimization of PID Controller Parameters for Fuel Cell Voltage and Fuel Utilization," Sustainability, vol. 11, no. 12, pp. 1-20, Jun. 2019.

[21] M. I. Solihin, L. F. Tack, and M. L. Kean, "Tuning of PID Controller Using Particle Swarm Optimization (PSO)," Int. J. Adv. Sci. Eng. Inf. Technol., vol. 1, no. 4, p. 458, 2011.

[22] F. S. M. Alkhafaji, W. Z. W. Hasan, M. M. Isa, and N. Sulaiman, "A novel method for tuning PID controller," J. Telecommun. Electron. Comput. Eng., vol. 10, no. 1-12, pp. 33-38, 2018.

[23] A. Triwiyatno, S. Sumardi, and E. Apriaskar, "Robust fuzzy control design using genetic algorithm optimization approach: case study of spark ignition engine torque control," Iran. J. Fuzzy Syst., vol. 14, no. 3, pp. 1-13, 2017. 